\title{
A comprehensive study of a wide energy range proportional counter neutron spectrometer
}

\author{
M. Benmosbah, J.E. Groetz* \\ LMN-AC (UMR CEA E4), Université de Franche-Comté, 16 route de Gray, 25030 Besançon \\ Cedex, France \\ Email.jegroetz@univ-fcomte.fr

\section{P. Crovisier} \\ SPR (UMR CEA E4), CEA Valduc, 21120 Is-sur-Tille, France
}

\section{B. Asselineau, L. Van-Ryckeghem}

LMDN, IRSN, 13115 St Paul lez Durance, France

\section{H. Truffert, A. Cadiou}

SPR, Cogéma La-Hague, 50444 Beaumont, France

\section{Ziarnowski}

DQS, CEA DIF, 91680 Bruyères le Chatel, France

The neutron spectrometer ROSPEC is made up of 6 spherical proportional counters with different gas fillings, on a rotating platform, and which covers the energy range $0.025 \mathrm{eV}$ to $4.5 \mathrm{MeV}$. A complete study is performed within the framework of a French working group in order to improve the spectrometer performance. First, the study of the devices performance in reference neutron fields was performed, with respect to the influence of rotation and disturbance from other counters. It appeared that the fluence scattered by other counters could represent between 7 and $40 \%$ of the total fluence, but with a slight influence on the initial spectrum. Secondly, we determined the response matrix for each counter from two main parameters, the wall and the gas amplification effects. The wall effects were calculated using the Snidow algorithm and compared to the MCNPX simulation. The change in the electric field was calculated using a finite element method to define amplification region for simulation. Finally, since the ROSPEC unfolding code sometimes gave negative fluences, the unfolding code UMG was used to correct this default. We compared the results from both unfolding codes.

International Workshop on Fast Neutron Detectors and Applications

April, 3-6, 2006

University of Cape Town, South Africa

* Speaker. 


\section{Introduction}

The French working group ROSPEC, bringing together the French users of the neutron rotative spectrometer called ROSPEC (manufactured by Bubble Technology industries, Ontario, Canada), performed a comparison in well-characterised neutron fields to test the spectrometric performances of three devices from the participant laboratories. The experiments have been carried out with the reference fields of ${ }^{252} \mathrm{Cf},\left({ }^{252} \mathrm{Cf}+\mathrm{D}_{2} \mathrm{O}\right) / \mathrm{Cd},{ }^{241} \mathrm{AmBe}$ and the SIGMA facility at the LMDN laboratory (Cadarache) from IRSN. The results [1] showed that for the sources with important thermal and epithermal parts (moderated ${ }^{252} \mathrm{Cf}$ and SIGMA), the measured values of the ambient dose-equivalent rate overestimate the reference between 20 and $40 \%$, while fluences in the bin 0.01 eV-50 keV are significantly overestimated in every case (within 40-235\%). Some discrepancies concerning fluence (negative values) exist close to $100 \mathrm{keV}$ for the SP2-1 counter.

Several queries occur from these results, concerning the influence of rotation, the response function of counters and the unfolding process.

\section{The ROSPEC spectrometer}

The ROSPEC spectrometer consists of six gas-filled proportional counters, designed to cover the energy range from $0.01 \mathrm{eV}$ to $4.5 \mathrm{MeV}$ [2, 3]. Three SP2 counters (diameter of $5.04 \mathrm{~cm}$, i.e. 2 inches) are filled with hydrogen under pressures of $76 \mathrm{kPa}$ (SP2-1, because initially the pressure was 1 atmosphere), $0.4 \mathrm{MPa}$ (SP2-4, that is $4 \mathrm{~atm}$.) and $1 \mathrm{MPa}$ (SP2-10, that is $10 \mathrm{~atm}$.) to measure the range from $50 \mathrm{keV}$ to $1.5 \mathrm{MeV}$. A SP6 counter (diameter of $15.24 \mathrm{~cm}$, i.e. 6 inches) is filled with an argon-methane mixture at $507 \mathrm{kPa}(1.2-4.5 \mathrm{MeV})$. Two counters are ${ }^{3} \mathrm{He}$ filled, one is bare (0.01-1 eV) and the other with a boron shell $(1 \mathrm{eV}-50 \mathrm{keV})$. These detectors are mounted on a circular base plate which rotates about a vertical axis at $4 \mathrm{rpm}$ to average out local variations in the neutron field. The unfolding of the proton-recoil pulse-height distributions from the counters is done sequentially using the software ZSPEC4.

\section{Overall response of the ROSPEC spectrometer}

\subsection{Effects of rotation on the neutron fluence in the counters}

Calculations of the influence of rotation for the 6 counters were performed using MCNPX code [4]. The geometry of the irradiation facility and the room scattering were taken into account. For each neutron field, the neutron fluence spectrum was determined in every counter by using its own neutron energy range. The rotation motion was taken into account and a mean spectrum was calculated by using 8 successive instantaneous positions for every counter. These 8 positions are characterized by an angle $\theta$ between 0 and $2 \pi$, i.e. with a step of $\pi / 4$. $\Phi_{\text {rotation }}$ is the average of the neutron fluence in the counter $i$ in its 8 positions:

$$
\left(\Phi_{\text {rotation }}\right)_{i}=\frac{1}{8} \sum_{\theta=0}^{\frac{7 \pi}{4}}\left(\Phi_{\theta}\right)_{i}
$$

In order to assess the rotation effects, the reference of the neutron fluence $\Phi_{\text {fixed }}$ is calculated for every counter as if it was located at the centre of the circular base plate. Thus, the ratio $r_{i}$, which 
expresses the influence of rotation on the counter $i$ is given by:

$$
r_{i}=\frac{\left(\Phi_{\text {rotation }}\right)_{i}}{\left(\Phi_{\text {fixed }}\right)_{i}}
$$

The results (Table 1) show that the influence of rotation on the neutron fluence is weak for most of the counters, excepted for the ${ }^{3} \mathrm{He}$ counter where this influence may reach $30 \%$. The SP6 counter is the less influenced due to its large size.

\begin{tabular}{|c|c|c|c|c|c|c|}
\hline Ratio $r_{i}$ & ${ }^{3} \mathrm{He}$ & ${ }^{3} \mathrm{He}+{ }^{10} \mathrm{~B}$ & $\mathrm{SP} 2-1$ & $\mathrm{SP}-4$ & $\mathrm{SP} 2-10$ & SP6 \\
\hline${ }^{252} \mathrm{Cf}$ & - & - & $0.97(0.05)$ & $1.03(0.04)$ & $1.03(0.04)$ & $1.00(0.01)$ \\
\hline$\left({ }^{252} \mathrm{Cf}+\mathrm{D}_{2} \mathrm{O}\right) / C d$ & $0.72(0.02)$ & $1.04(0.09)$ & $0.93(0.09)$ & $1.02(0.08)$ & $1.04(0.08)$ & $1.02(0.02)$ \\
\hline${ }^{241} \mathrm{AmBe}$ & - & - & $0.95(0.08)$ & $1.03(0.05)$ & $1.03(0.03)$ & $1.00(0.02)$ \\
\hline
\end{tabular}

Table 1: Ratio $r_{i}$ showing the influence of rotation for every counter in relation to the neutron source, with the relative error in brackets.

\subsection{Influence of rotation and scattering from other counters}

It was possible to calculate, for every counter, the influence of both rotation and neutron fluence scattered by the five other counters. Thus, one can deduce for a given counter $i$ its total neutron fluence and the proportion of its fluence which is due to rotation and scattering by the other counters, referred as $S_{i}$ :

$$
S_{i}=\frac{\Phi_{\text {rospec }}}{\Phi_{\text {counter i }}}
$$

\begin{tabular}{|c|c|c|c|c|c|c|}
\hline Ratio $S_{i}$ & ${ }^{3} \mathrm{He}$ & ${ }^{3} \mathrm{He}+{ }^{10} \mathrm{~B}$ & $\mathrm{SP} 2-1$ & $\mathrm{SP}-4$ & $\mathrm{SP} 2-10$ & SP6 \\
\hline${ }^{252} \mathrm{Cf}$ & - & - & $0.97(0.02)$ & $0.98(0.01)$ & $0.93(0.03)$ & $0.99(0.01)$ \\
\hline$\left({ }^{252} \mathrm{Cf}+\mathrm{D}_{2} \mathrm{O}\right) / C d$ & $0.57(0.02)$ & $0.93(0.04)$ & $0.94(0.05)$ & $0.94(0.08)$ & $0.99(0.05)$ & $0.99(0.01)$ \\
\hline${ }^{241} \mathrm{AmBe}$ & - & - & $0.95(0.08)$ & $1.03(0.05)$ & $1.03(0.03)$ & $0.99(0.01)$ \\
\hline
\end{tabular}

Table 2: Ratio $S_{i}$ showing the influence of rotation and scattering from the five other counters on every counter, with the relative error in brackets.

Calculations show that the influence of rotation and scattering is more or less significant according to the size and the relative position of every counter. SP6 has the weakest interference from the other counters due to its large size, while ${ }^{3} \mathrm{He}$ counter is highly perturbed. Several factors account for these results: the neutron energy through cross sections, the counter geometry through its diameter and its solid angle of detection, the relative position of every counter through the possibility of shielding by the other ones.

\section{Response function of the proportional counters}

\subsection{Wall effects and proton recoil spectra}

In the energy range between $50 \mathrm{keV}$ and a few $\mathrm{MeV}$, neutrons scatter elastically only. Provided that all the protons are stopped in the detector volume, for incident monoenergetic neutron of 
energy $E_{n}$, the energy probability density distribution for the recoil protons is rectangular. However, protons cannot deposit their entire energy if they hit the counter wall before being completely stopped, due to their range comparable to the counter dimensions. The Snidow algorithm [5] gives an analytical method for calculating the theoretical response function, taking into account the wall effect for a spherical counter without dead region. In the present work, the analytical response functions were calculated for the SP2-1, SP2-4, SP2-10 and SP6 counters, in their region of interest (ROI) for the recoil proton energy range. To provide accurate analytical response functions, the proton ranges have been calculated using the SRIM code [6] for each energy bin of the considered $R O I$ and the corresponding uncertainties have been evaluated.

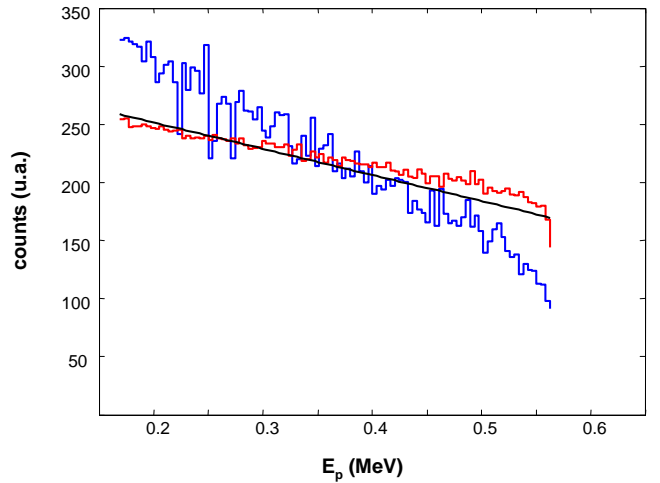

a)

Figure 1: Proton recoil spectra obtained with monoenergetic neutrons (blue), calculated with MCNPX (red) and with the Snidow algorithm (black): a) SP2-4 with neutrons at $565 \mathrm{keV}$. b) SP6 with neutrons at $4.5 \mathrm{MeV}$.

From Figure 1, the MCNPX calculation and the Snidow algorithm are in strong accordance for the hydrogenated counters (Figure 1a), but differ highly for a gas mixture at high energy (Figure 1b). The main reason should be a difference between the proton range data which are used. The MCNPX results and measurements are closely related for the SP6 counter. Differences between both MCNPX and experimental data are due to a low statistic of counts per channel and the electric field effects.

\subsection{Electric field effects}

A special design for the spherical proportional counter was proposed, in order to have the electric field constant along the anode wire [7]. Nevertheless, the wire holders and polar tubes alter the electric field in their vicinity, so that the gas amplification is reduced in this region. A previous study was done [8], using the method of virtual electric charges for solving the Laplace equation. We performed calculations of the electric field and the field line inside the counter with a finite elements method. A further work should be the definition of regions for the gas amplification in the MCNPX code. This should yield to the most accurate response function for every counter. 


\section{Unfolding process}

\subsection{Unfolding with ZSPEC4}

The unfolding code ZSPEC4 is used for the deconvolution of every counter sequentially and for merging the spectra calculated from the various counters [9]. $A_{j}$ is the number of counts (pulse height) in the energy group $j$ :

$$
A_{j}=\sum_{i=1}^{M}\left(\Phi_{n}\right)_{i} R_{i j}
$$

where $\left(\Phi_{n}\right)_{i}$ is the neutron fluence in $i$ and $R_{i j}$ is a triangular matrix of recoil proton response in $j$ by incident neutron in the energy group $i$, for $M$ energy groups. The response function is calculated from the Snidow algorithm. From the equation 5.1, the neutron fluence in the energy group $E=E_{\max }$ is:

$$
\left(\Phi_{n}\right)_{j}=\frac{A_{M}}{R_{M M}}
$$

The neutron fluence in the other energy groups is obtained by an iterative subtraction of counts due to neutrons in the upper energy group, such as:

$$
\left(\Phi_{n}\right)_{j}=\frac{A_{j}-\sum_{i=j+1}^{M}\left(\left(\Phi_{n}\right)_{j} R_{i j}\right)}{R_{i j}}
$$

Since the SP2-1 counter is the last one of this iterative process, negative solutions for fluence are possible due to the propagation of uncertainties [1, 10].

\subsection{Unfolding with UMG}

The UMG package is made up of two codes, MAXED and GRAVEL [11]. MAXED applies the maximum entropy principle to the unfolding of neutron spectrometric measurements [12]. The solution spectrum $\Phi_{i}$ that maximizes the entropy of the distribution is:

$$
\Phi_{i}=\Phi_{i}^{D E F} \exp \left[-\sum_{k} \lambda_{k} R_{k i}\right]
$$

where $\Phi_{i}^{D E F}$ is a default spectrum that contains the a priori information, $R_{k i}$ is the response function for the detector $k$ at the energy $i$ and $\lambda_{k}$ is a calculated output parameter. The default spectrum could be a flat spectrum or the spectrum of the known source.

The unfolding of neutron measurements with MAXED were performed for every counter in its detection range and compared with results from ZSPEC4.

Figure $2 \mathrm{a}$ displays a solution spectrum from UMG that fits the ISO data when the default spectrum is the known source, with a non negative values for fluence, contrary to ZSPEC4. But the solution spectrum is not accurate with a flat default spectrum. In this case, especially for the $\mathrm{D}_{2} \mathrm{O}$ moderated ${ }^{252} \mathrm{Cf}$ (Figure $2 \mathrm{~b}$ ), the solution spectrum is monotone and does not show the neutron resonance on ${ }^{16} \mathrm{O}$ at $435 \mathrm{keV}$ and $1 \mathrm{MeV}$.

Thus, unfolding using MAXED with the source spectrum as the default spectrum yields better results than ZSPEC4. 

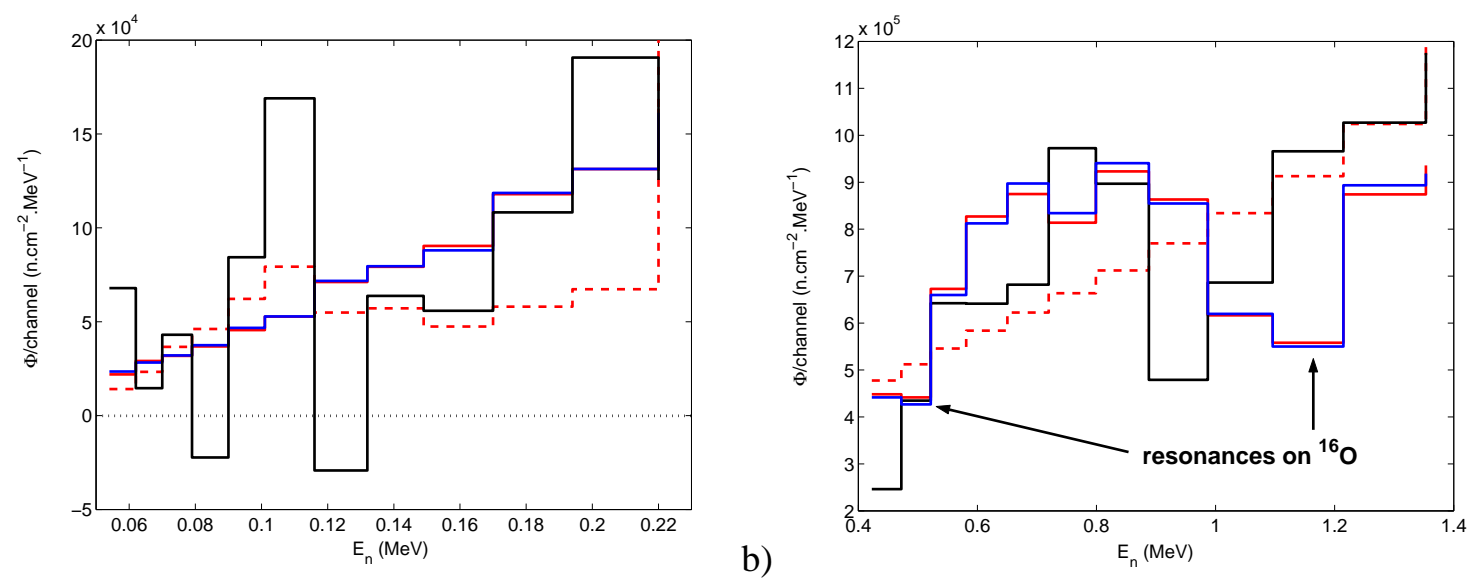

Figure 2: Unfolding of counter measurements using UMG with source spectrum as default (red), using UMG with flat spectrum as default (red dashed), using ZSPEC4 (black) and the correponding ISO source spectrum (blue) a) Unfolding of SP2-1 with ${ }^{252} \mathrm{Cf}$. b) Unfolding of SP2-10 with ${ }^{252} \mathrm{Cf}+\mathrm{D}_{2} \mathrm{O}$.

\section{Conclusion}

Influence of counter rotation and scattering from other counters have been calculated, showing that it depends on the size and the relative position of every counter. The calculation of a more accurate response function yields to better results with the unfolding using UMG. Further works will consist in taking into account the electric field effects to get the most accurate response function and in merging the neutron spectra from the six counters.

\section{References}

[1] P. Crovisier, B. Asselineau, G. Pelcot, L. Van-Ryckeghem, A. Cadiou, H. Truffert, J.E. Groetz and M. Benmosbah, French comparison exercise with the rotating neutron spectrometer ROSPEC, Radiat. Prot. Dosim., 115 (1-4) (2005) 324.

[2] H. Ing, W.G. Cross, P.J. Bunge, Spectrometers for radiation protection at Chalk River nuclear laboratories, Radiat. Prot. Dosim. 10 (1-4) (1985) 137.

[3] H. Ing, T. Clifford, T. Mc Lean, W. Webb, T. Cousins, J. Dhermain, ROSPEC - A simple reliable high resolution neutron spectrometer, Radiat. Prot. Dosim. 70 (1-4) (1997) 273.

[4] D.B. Pelowitz, MCNPX Version 2.5.0, Los Alamos National Laboratory Report LA-CP-05-0369 (2005).

[5] N.L. Snidow, H.D. Warren, Wall effect corrections in proportional counter spectrometers, Nucl. Instr. and Meth. 51 (1967) 109.

[6] J.F. Ziegler J.P. Biersack, SRIM Code, 2003.

[7] P.W. Benjamin, C.D. Kemshall and J. Redfearn, A high resolution spherical proportional counter, Nucl. Instr. and Meth. 59 (1968) 77.

[8] K. Weise, M. Weyrauch and K. Knauf, Neutron response of a spherical proton recoil proportional counter, Nucl. Instr. and Meth. A 309 (1991) 287. 
[9] J.C. Nunes and R.T. Faught, Commisioning of a proton-recoil spectrometer, Radiat. Prot. Dosim. 87 (4) (2000) 251.

[10] M.W. Mallett, T.D. McLean, R.H. Olsher, L.L Romero, D.T. Seagraves, R.T. Devine, S.L. Eisele, J.M. Hoffman and R.O. Murphy, Multisphere default spectra-solution spectrum and dosemeter response implications, Radiat. Prot. Dosim. 110 (2004) 549.

[11] B. Wiegel, M. Reginatto, UMG package 3.3, PTB Braunschweig (2004).

[12] M. Reginatto, P. Goldhagen, S. Neumannn, Spectrum unfolding, sensitivity analysis and propagation of uncertainties with the maximum entropy deconvolution code maxed, Nucl. Instr. and Meth. A 476 (2002) 242. 